\title{
Application of Improved Cohen Bilinear Time-frequency Distribution for Doppler Motion Feature Extraction
}

\author{
Xi Wang ${ }^{1, \text { a }}$, Zhuo Chen ${ }^{1, \mathrm{~b}}$, JiaWu He $\mathrm{e}^{2, \mathrm{c}}$ and Gang Zhu ${ }^{1, \mathrm{~d}}$ \\ ${ }^{1}$ Department of Information Engineering, Academy of Armored Forces Engineering, Beijing, China \\ ${ }^{2}$ Department of Scientific Research, Academy of Armored Forces Engineering, Beijing, China \\ lycis@126.com, ${ }^{\mathrm{b}}$ ellen nehc@yahoo.com, 도iawu he@126.com, ${ }^{\mathrm{d}}$ 18901078269@189.cn
}

Keywords: Doppler motion; feature extraction; human gait; time-frequency distribution.

Abstract. Doppler signals of body movement are measured and collected by using X-band continuous wave radar to extract the human motion characteristics of radar echo. In order to suppress the cross terms more effective and retain the high resolution time frequency resolution, This paper construct a window function to the kernel function of Cohen bilinear time-frequency distribution, thus obtain a improved type of Cohen time-frequency distribution. We achieve good result in extraction of micro Doppler frequency characteristics of human gait.

\section{Introduction}

Using radar to detect human motion, the echo contains the Doppler frequency information generated by the human motion modulation. The human body is in a non-uniform rigid body motion state, the Doppler echo is time-varying, the body of the body of the different movements of the body will make its echo spectrum has a side lobe or broaden[1]. This phenomenon is called the micro Doppler Effect. According to the definition of the Navy Research Laboratory, the micro motion is part of the target or targets in addition to the mass translation vibration, rotational, and accelerated motion. Micro Doppler features are described from the frequency of the target micro motion characteristics. Human body movement characteristics include the movement of human body, the body's rotation, swing, etc. The detection of human motion characteristics of radar can judge the motion state of the human body static targets, and walk and run, and can be used in battlefield surveillance, security door, biomechanical and kinematic studies. For example, human gait patterns hands and legs swing the echo signal Doppler frequency shift, the incentive of micro Doppler frequency approximate sinusoidal modulation, this unique modulation by the amplitude and frequency of the two parameters can be described. Using time-frequency analysis technique to extract the micro Doppler features, it can effectively estimate the motion law of the human body. At present, the research on the feature extraction of human motion radar is mainly based on the measured data, and the micro Doppler motion characteristics of the human body are extracted by using non-stationary signal processing method[2,3].

\section{Acquisition Micro Doppler Radar Echo of Human Motion}

In target speed in the same conditions, the shorter wavelength radar Doppler frequency produced by more and more conducive to extract the target speed change minor character and therefore high band is more conducive to human motion characteristic extraction. In this paper, we use the $\mathrm{X}$ - band radar, and the working frequency is $10.5 \mathrm{GHz}$, and the transmission power is $200 \mathrm{~mW}$. The height of the human body is 1.70 meters, including walking, running, crawling three kinds of motion state. 

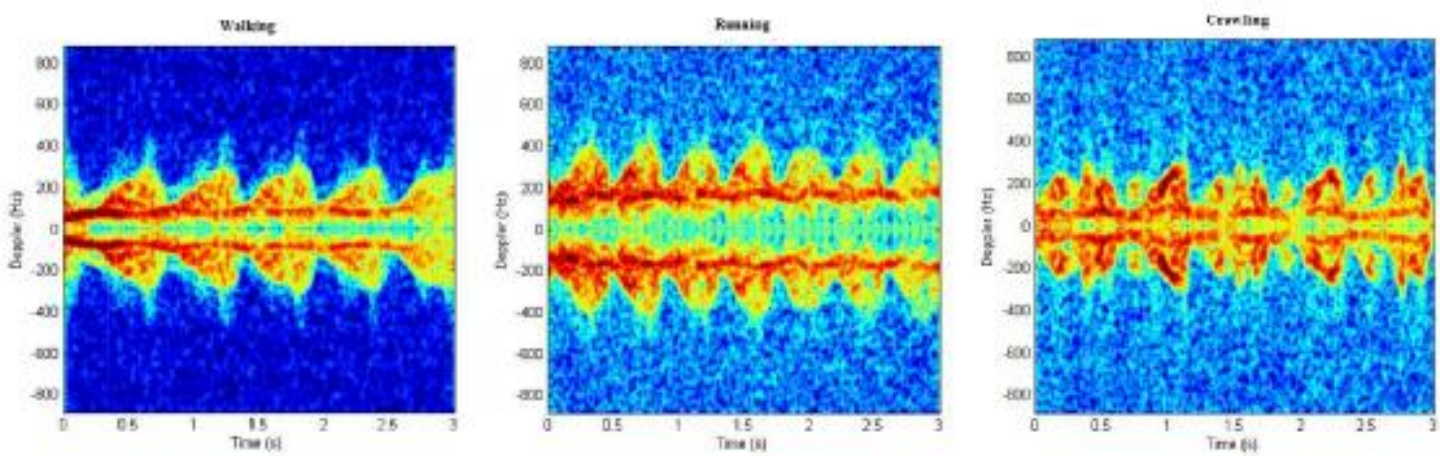

Fig. 1. Doppler frequency of human gait

Micro Doppler estimation target motion parameter of the premise, extracting micro Doppler of the key lies in the micro Doppler radar echo signal instantaneous frequency is estimated with high precision, need to focus on breakthrough has high time-frequency resolution of time-frequency distribution[2] and its efficient computation.

\section{Feature Extraction of Human Gait based on Wigner-Ville distribution}

In 1948, J.Ville introduced the Wigner distribution of E.P.Wigner in 1932, and many scholars have followed suit. After nearly half a century of development, the time frequency distribution of the theory, or in the engineering application, have become mature [98]. The Cohen bilinear time-frequency distribution estimation, the essence of which is the signal energy distribution in frequency plane, based on Wigner Ville distribution, the distribution is proposed by Wigner in the study of quantum mechanics, this distribution can be expressed as follows[3]:

$$
V D_{S}(t, \omega)=\int s\left(t+\frac{\tau}{2}\right) s *\left(t-\frac{\tau}{2}\right) e^{-j \omega t} d \tau
$$

In the formula (1), the signal $\mathrm{s}(\mathrm{t})$ appears 2 times, without any window function, which avoids the linear time frequency distribution such as short time Fourier transform (STFT) in time and frequency resolution in each other to contain Wigner Ville distribution is not linear, i.e. two signals and the Wigner Ville distribution is not every signal Wigner Ville distribution and, out of which more than an additional term. The third one is the cross terms of the two. Cross is real and mixed in between, and its magnitude is two times, cross terms in the presence of serious interference with people understanding of Wigner Ville distribution, especially when the signal becomes complex, Wigner Ville distribution will become meaningless[4].
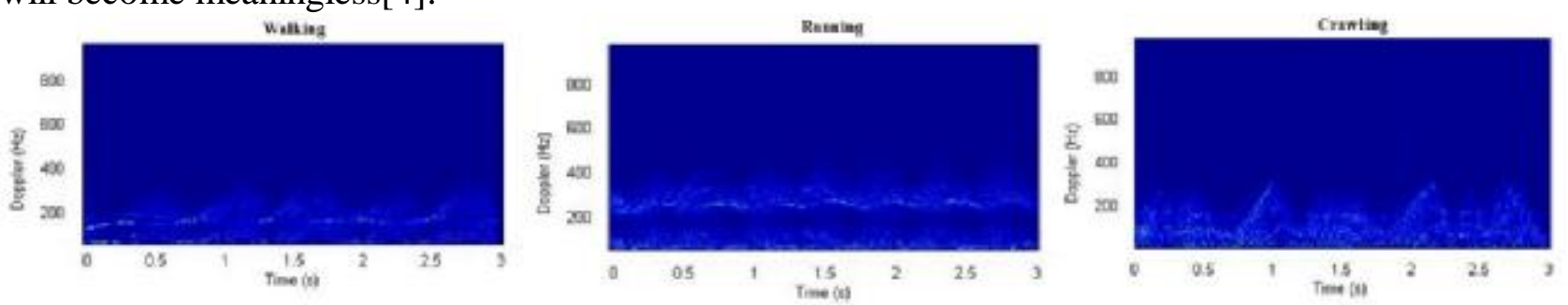

Fig. 2. Wigner-Ville distribution of human gait

\section{Improved Cohen Bilinear Time-frequency Distribution}

Although the distribution of Wigner-Ville is attractive, its application is limited. In 1966, time-frequency analysis of Cohen Leon proposed a new time-frequency distribution of Wigner-Ville distribution, and then constructed a new time-frequency distribution method. The method can be expressed as:

$$
\operatorname{TFR} s(t, f)=\iint \Phi(\tau, \theta) W V D s(t-\tau, f-\theta) d \tau d \theta
$$

Where $\Phi(\tau, \theta)$ is kernel function. The work of Cohen has the milestone significance. By choosing different kernel functions, the time frequency distribution of the desired characteristics can be 
generated. Cohen is used to smooth the Wigner-Ville distribution in order to suppress the cross terms of Wigner-Ville distribution, and it is the cost of the whole distribution. According to the definition of Cohen, the time-frequency distribution can be expressed as a bilinear time frequency distribution.

$$
P_{x}(t, f ; \phi)=\frac{1}{2 \pi} \iiint e^{-j(\theta t+\omega t-\mu \theta)} \phi(\tau, \theta) x\left(\mu+\frac{\tau}{2}\right) x *\left(\mu-\frac{\tau}{2}\right) d u d \tau d \theta
$$

By choosing different kernel functions, the time-frequency distribution of different performances can be obtained. The convolution of the Fourier Transform of the time domain multiplication of the functions corresponding to the Fourier Transform (3) can be written as:

$$
P_{x}(t, f ; \phi)=\left(\frac{1}{2 \pi}\right)^{2} \iint \Phi(t-\tau, \omega-\theta) W_{x}(\tau, \theta) d \tau d \theta
$$

Where $\Phi(\mathrm{t}, \omega)$ as the kernel function $\phi(\tau, \theta)$ of the two-dimensional Fourier Transformation.

$$
\Phi(t, \omega)=\iint \phi(\tau, \theta) e^{-j(\theta t+\omega \tau)} d \theta d \tau
$$

It is known from $(5), P_{x}(t, f ; \phi)$ understanding for the two-dimensional convolution of the signal WVD and the kernel function of the WVD, convolution is equivalent to the filtering operation. The formula (4) expressed as below.

$$
\begin{aligned}
& P_{x}(t, f ; \phi)=\frac{1}{2 \pi} \int_{-\infty}^{+\infty} \int_{-\infty}^{+\infty} e^{-j(\theta t+\omega \tau)} \phi(\tau, \theta)\left[\int_{-\infty}^{+\infty} x\left(\mu+\frac{\tau}{2}\right) x^{*}\left(\mu-\frac{\tau}{2}\right) e^{j \mu \theta} d \mu\right] d \tau d \theta \\
& P_{x}(t, f ; \phi)=\frac{1}{2 \pi} \int_{-\infty}^{+\infty} \int_{-\infty}^{+\infty} e^{-j(\theta t+\omega \tau)} \phi(\tau, \theta) A_{x}(-\theta, \tau) d \tau d \theta
\end{aligned}
$$

In the fuzzy domain, the signal energy concentrated near the origin and cross energy will move away from the origin, through in ambiguity domain proper .By selection of kernel function $\phi(\tau, \theta)$, Cohen bilinear time-frequency distribution retain only the signal and eliminate the cross terms, thus we can obtain high time-frequency resolution time frequency distribution. In the time-frequency domain, Cohen bilinear time-frequency distribution is the result of kernel function of two-dimensional Fourier transform of the Wigner Ville distribution for smooth, smooth the Cohen bilinear time-frequency distribution of the signal spreading. When the frequency resolution reduce, sometimes even lose some good performance. A wide of the smoothing function, the ambiguity domain is a narrow weighted window. At this time the window function can better suppress the cross terms, makes Cohen bilinear time-frequency distribution of signals is not focused, on the contrary, a wide of the smoothing function, the ambiguity domain is a wide window weighted, it makes Cohen bilinear time frequency distribution of signal is more concentrated, but inhibited cross ability is poor. Therefore, the selection of smoothing functions needs to be a compromise between the suppression of the cross term and the improvement of the resolution[5].

In order to more effective for suppressing the cross terms, at the same time as possible to retain the high resolution time frequency resolution, this paper in Cohen bilinear time-frequency distribution based on kernel function were windowed processing, thus obtained for a class of improved type of Cohen class time-frequency distribution[6]. we refer to as the core and window Cohen bilinear time-frequency distributions. Through to kernel windowing and Cohen class time-frequency distribution ability to suppress cross terms has been greatly improved, at the same time frequency resolution has been retained as far as possible, because can change the shape of the window function, so that the energy of the kernel function in fuzzy domain concentrated in the neighborhood of the origin, so as to improve the cross term suppression ability. Signal $s(t)$ of the nuclear and Cohen type bilinear time-frequency distribution can be expressed as follows.

$$
P_{x}(t, f ; \phi)=\frac{1}{2 \pi} \iiint[g(\theta) \phi(\theta, \tau) h(\tau)] s\left(\mu+\frac{\tau}{2}\right) s *\left(\mu-\frac{\tau}{2}\right) e^{-j(\theta t+\omega \tau-\mu \theta)} d \mu d \tau d \theta
$$

Among them, $g(\theta)$ and $h(\tau)$ are respected as in frequency domain and time domain smoothing window. Before window processing of kernel function $\phi(\theta, \tau)=e^{-\frac{\theta^{2} \tau^{2}}{\sigma}}$ (where $\left.\sigma>0\right)$, the fuzzy domain 
energy of kernel function distribution is more dispersed. After window processing of kernel function, the result is shown as below.
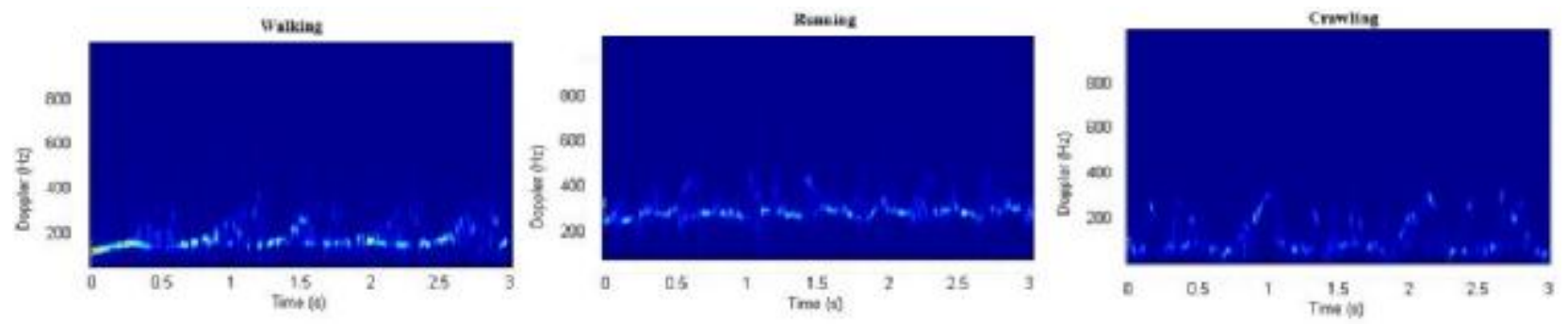

Fig. 3. Improved Cohen Bilinear distribution of human gait

\section{Conclusion}

As shown in fig.3, kernel function in fuzzy domain energy will be concentrated in the vicinity of the origin, so kernel windowed Cohen class time-frequency distribution has better cross term suppression ability. Selecting a proper window function to the weighted kernel function, then processing two-dimensional filtering of the Wigner Ville distribution of signal $s(t)$, we can effectively suppress the cross terms, and be as far as possible to retain the Wigner Ville distribution of the advantages of high frequency resolution. In the ambiguity domain, the signal energy concentrated near the origin and cross energy will move away from the origin, through in the ambiguity domain proper window function of kernel function $\phi(\theta, \tau)$ are weighted, Cohen bilinear time-frequency distribution in fuzzy domain energy completely distributed near the origin, in order to inhibit cross terms. In practice, we can use the window function including Hamming window, Hanning window, Bartlett window, Blackman window, Gauss window and Kaiser window.

\section{References}

[1] Otero M. Application of a continuous wave radar for human gait recognition. SPIE Proceed-ings on Signal Processing, Sensor Fusion, and Target Recognition, (5809):538--548 (2005)

[2] Chen V C. Micro-Doppler effect of micro-motion dynamics: a review. Proceedings of SPIE on Independent Component Analyses, Wavelets, and Neural Networks, 240--249 (2003).

[3] Chen V C, Li F. Analysis of micro-Doppler signatures. J. IEEE Proceedings on Radar, Sonar and Navigation. 150(4): 271--276 (2003).

[4] Sejdic E, Djurovic I, Jiang J. Time-frequency feature representation using energy concentration: an overview of recent advances. J. Digital Signal Processing, (19):153--183 (2009).

[5] Boashash B, Ristich B. Polynomial Wigner-Ville distributions and time-varying higher-order spectra. Victoria: Proceedings of the IEEE-SP International Symposium on Time-Frequency and Time-Scale Analysis, 31--34 (1992).

[6] Boashash B, O'Shea P. Polynomial Wigner-Ville distributions and their relationship to time-varying higher order spectra. J. IEEE Transactions on signal processing, 42(1):216--220 (1994). 